\title{
A HOMOGENEOUS CONTINUUM THAT IS NON-EFFROS
}

\author{
DAVID P. BELLAMY AND KATHRYN F. PORTER
}

(Communicated by James E. West)

\begin{abstract}
Using a very geometric, intuitive construction, an example is given of a homogeneous, compact, connected Hausdorff space $(X, T)$ that does not satisfy the conclusion of the Effros Theorem. In particular, there is a point $p$ and a neighborhood $V$, of the identity in the group of self-homeomorphisms on $X$, with the compact-open topology such that $V_{p}=\{h(p): h \in V\}$ is nowhere dense in $X$.
\end{abstract}

\section{INTRODUCTION}

A topological space $X$ is homogeneous provided that for every $x, y \in$ $X$ there exists a homeomorphism $h: X \rightarrow X$ such that $h(x)=y$. Let $X$ be a homogeneous Hausdorff space and let $H(X)$ be its group of selfhomeomorphisms. Let $x \in X$ and let $E_{x}: H(X) \rightarrow X$ denote the map defined by $E_{x}(h)=h(x)$. If $X$ is a compact metric space and $H(X)$ is given the standard supremum metric then E. G. Effros [4] has shown that $E_{x}$ is an open mapping for each $x \in X$. (Actually this is only a very special case of what Effros proved.) The second author [6] has shown that this also holds for arbitrary products of homogeneous compact metric spaces and has introduced a concept called even homogeneity, which is useful in studying different versions of this question. We are not giving a definition of even homogeneity here because it is not needed in our argument. In this paper, a counterexample to two other proposed generalizations of this result is presented.

Let $X$ and $Y$ be topological spaces and let $F$ be a family of functions from $X$ to $Y$. Let $A \subset X$ and $B \subset Y$. Then $(A, B)$ denotes $\{f \in F: f(A) \subset B\}$. The compact-open topology on $F$ has as a subbase $\{(A, B): A \subset X, B \subset Y, A$ is compact, and $B$ is open $\}$. The set $\{(A, B): A \subset X, B \subset Y, A$ is closed, $B$ is open, and either $A$ or $(Y \backslash B)$ is compact $\}$ is a subbase for the complemented compact-open topology on $F$. We point out that if $X$ is compact then these

Received by the editors February 20,1990; presented by the first author at the Twenty-Third Annual Spring Topology Conference, March 1989, University of Tennessee, Knoxville, Tennessee.

1980 Mathematics Subject Classification (1985 Revision). Primary 54C35, 57S05; Secondary $54 \mathrm{H} 13$.

Key words and phrases. Homogeneous, Effros' Theorem, compact-open topology, continuum, monotone map, group of self-homeomorphisms, complemented compact-open topology. 
two topologies on $F$ coincide. Also the compact-open topology is the same as that induced by the supremum metric mentioned above, in the case where $X$ is compact metric and $Y$ is metric.

In [2] R. Arens showed that if $X$ is a locally compact Hausdorff space and $H(X)$ is given the complemented compact-open topology, then $H(X)$ is a topological group. By the remark in the last paragraph, this is also true if $X$ is compact Hausdorff and $H(X)$ is given the compact-open topology. Ancel has asked whether the conclusion of the Effros theorem is true (i.e., the maps $E_{x}$ are open) if either $X$ is homogeneous, compact, Hausdorff and $H(X)$ is given the compact-open topology, or $X$ is homogeneous, locally compact, Hausdorff and $H(X)$ is given the complemented compact-open topology. We answer both these questions in the negative by providing a counterexample to the compact case.

A continuum here means a compact, connected, Hausdorff space. A map $f: X \rightarrow Y$ is monotone provided that for each $y \in Y, f^{-1}(y)$ is a continuum. It is well known (and easy to prove) that this implies that $f^{-1}(M)$ is a continuum for each continuum $M \subset Y$, in the case that $X$ is compact and $f$ is surjective.

If $A \subset H(X)$ and $x \in X, A x$ denotes the set $\{h(x): h \in A\}$. Similarly, if $B \subset X$, then $A B=\{h(x): h \in A, x \in B\}$. A subgroup $G$ of $H(X)$ is called transitive provided that for each $x \in X, G x=X$.

\section{THE EXAMPLE}

As is customary, let $S^{2}=\left\{x \in \mathbf{R}^{3}:\|x\|=1\right\}$.

If a single point is removed from $S^{2}$, and in its place a circle is attached nicely, the resulting space is a disk. Doing the same thing at two points yields an annulus. If this process is repeated at each point of a countable dense subset of $S^{2}$, the result is the Sierpinski universal plane curve. The example in this paper can be thought of as the space obtained when this process of removing a point and inserting a simple closed curve in its place is carried out at every point of $S^{2}$.

Formally, it is convenient to think of this space as a subset of $S^{2} \times S^{2}$ with a specially constructed topology. Define $X=\left\{(x, y) \in S^{2} \times S^{2}: x \cdot y=0\right\}$, where $x \cdot y$ denotes the usual vector dot product of $x$ and $y$. Thus, $(x, y) \in X$ if and only if $y$ is on the equator of $S^{2}$ relative to $x$ as a pole.

For any $x \in S^{2}$, let $S^{1}(x)=\left\{y \in S^{2}: x \cdot y=0\right\}$. The circle of points replacing the point $x \in S^{2}$ is just going to be $\{x\} \times S^{1}(x)$. For any $x, p \in S^{2}$, if $p \neq \pm x$, define $p(x \perp)=(p-(x \cdot p) x) /\|p-(x \cdot p) x\|$. Thus $p(x \perp)$ is the normalized projection of $p$ perpendicular to $x$. In particular, $p(x \perp) \in S^{1}(x)$.

The topology on $X$ is defined as follows: Each $\{x\} \times S^{1}(x)$ will have its usual topology. Let $x \in S^{2}$, let $U$ be any open set in $S^{1}(x)$, and let $0<\varepsilon<2$. The set $B(x, U, \varepsilon)$ is the set $B_{1} \cup B_{2}$, where $B_{1}=\{(x, p): p \in U\}$, while $B_{2}=\{(p, q): p(x \perp) \in U$ and $0<d(x, p)<\varepsilon\}$. (Here $d$ is the ordinary 
Euclidean distance.) Note that the definition of $B_{2}$ makes no use of the second coordinate. Thus, whenever $(p, q) \in B_{2},\{p\} \times S^{1}(p) \subset B_{2}$, i.e., $B_{2}$ is saturated with respect to the collection of simple closed curves $\left\{\{y\} \times S^{1}(y): y \in S^{2}\right\}$. The collection $\left\{B(x, U, \varepsilon): x \in S^{2}, U\right.$ open in $S^{1}(x)$, and $\left.0<\varepsilon<2\right\}$ is a base for our topology $T$ on $X$.

Intuitively, each point $p$ of $S^{1}(x)$ represents a direction (on $S^{1}(x)$ and in $X)$ from $x$. The point $(x, p)$ lies in the direction $p$ from $x$, as do all points $(t, q)$ for which $t(x \perp)=p$, regardless of what $q$ is. The basic open set $B(x, U, \varepsilon)$ then consists of all points whose direction from $x$ lies in the set $U$, and whose first coordinate is within $\varepsilon$ of $x$. Thus, $(x, p)$ is the "nearest" point in $X$ to $x$ lying in the direction $p$ from $x ; x$ itself, of course, does not belong to $X$ having been replaced by the circle $\{x\} \times S^{1}(x)$. Thus, the restriction $\varepsilon<2$ above is needed since the direction from $x$ to $y$ on $S^{2}$ doesn't mean anything if $x$ and $y$ are antipodal.

Define $F: X \rightarrow S^{2}$ by $F(x, y)=x$. Then for each $x \in S^{2}, F^{-1}(x)=$ $\{x\} \times S^{1}(x) . F$ is continuous since if $V$ is the disk about $x$ of radius $\varepsilon$, then $F^{-1}(V)=B\left(x, S^{1}(x), \varepsilon\right)$. By the saturated part of a set $G \subset X$, we mean the set $F^{-1}\left(S^{2} \backslash F(X \backslash G)\right)=\left\{(y, p) \in G:\{y\} \times S^{1}(y) \subset G\right\}$, the largest subset of $G$ saturated with respect to the collection $\left\{F^{-1}(x): x \in S^{2}\right\}$. In the case that $U$ is a proper nonempty open set in $S^{1}(x)$, the saturated part of $B(x, U, \varepsilon)$ is just the set $B_{2}$, defined above. Since every nonempty basic open set has nonempty saturated part, the saturated part of any nonempty open set $O$ is dense in $O$. This fact will be important later.

\section{Results about the eXAMPLe, $X$}

Lemma 1. $X$ is compact.

Proof. Let $\mathscr{U}$ be any open cover of $X$ by basic open sets. For each $x \in S^{2}$ define a finite subcollection $\mathscr{U}(x)$ of $\mathscr{U}$ covering $\{x\} \times S^{1}(x)=F^{-1}(x)$ as follows: If there exists $V_{x} \in \mathscr{U}$ such that $F^{-1}(x) \subset V_{x}$, then let $\mathscr{U}(x)=\left\{V_{x}\right\}$. If there is no such $V_{x} \in \mathscr{U}$, then let $\mathscr{U}(x)=\left\{V_{1}, V_{2}, \ldots, V_{n(x)}\right\}$ be a finite collection of elements of $\mathscr{U}$ covering the compact set $F^{-1}(x)$, with $n(x)$ as small as possible. Necessarily, each $V_{i}$ is of the form $B\left(x, O_{i}, \varepsilon_{i}\right)$ in this case. In either case, $F(\bigcup \mathscr{U}(x))$ is a neighborhood of $x$ in $S^{2}$. (In the first case, $\{x\} \times S^{1}(x)$ lies in the saturated part of $V_{x}$. In the second case, $\bigcup \mathscr{U}(x)$ is a saturated open set.) Consequently, the set $\left\{\operatorname{Int} F(\bigcup \mathscr{U}(x)): x \in S^{2}\right\}$ is an open cover of $S^{2}$ and so has a finite subcover, say $\left\{\operatorname{Int} F\left(\bigcup \mathscr{U}\left(x_{j}\right)\right): j=\right.$ $1,2, \ldots, m\}$. Now, let $\Upsilon=\bigcup_{j=1}^{m} \mathscr{U}\left(x_{j}\right) . \Upsilon$ is a finite union of finite sets and hence is finite. Let $(y, q) \in X$. Then $y \in \operatorname{Int} F\left(\bigcup \mathscr{U}\left(x_{j}\right)\right)$ for some $j$. Thus, $\{y\} \times S^{1}(y) \subset \bigcup \mathscr{U}\left(x_{j}\right)$. Therefore, $(y, q) \in \bigcup \mathscr{U}\left(x_{j}\right) \subset \bigcup \Upsilon$, so that $\Upsilon$ covers $X$, and the proof is complete.

The next lemma is striking in that it would not be possible in the compact metric case if all point inverses were nondegenerate. 
Lemma 2. Let $D_{x}$ denote the decomposition of $X$ whose nondegenerate elements are the sets $\{y\} \times S^{1}(y)$ for $y \neq x$. Let $F_{x}: X \rightarrow X / D_{x}$ be the quotient map. Then, $D_{x}$ is upper-semicontinuous for each $x$, and each $X / D_{x}$ is homeomorphic to a closed disk.

Proof. Use "suspension" coordinates on $S^{2}$, with $x$ as the upper vertex. Thus $(p, z)$ is a point on $S^{2}$, where $p$ is a point on $S^{1}(x)$ and $z$ is the $z$ coordinate computed as though $x$ were the point $(0,0,1)$. Define $H_{x}: S^{2} \backslash\{x\} \rightarrow S^{2}$ by $H_{x}(p, z)=(p,(z-1) / 2)$. Thus, the great semicircle from $-x$ to $x$ through $p$ is shrunk to the quarter circle from $-x$ to $p$. Then define $f_{x}: X \rightarrow S^{2}$ by

$$
f_{x}(s, t)= \begin{cases}H_{x}(s) & \text { if } s \neq x \\ t & \text { if } s=x\end{cases}
$$

It is easy to check that the point inverses of $f_{x}$ are the same as those of $F_{x}$, and the image $f_{x}(X)$ is the closed lower hemisphere of $S^{2}$ (relative to $x$ as $(0,0,1)$.) Continuity of $f_{x}$, and hence upper-semicontinuity of the collection $D_{x}$, are easily verified and therefore, are left to the reader.

Lemma 3. $X$ is a continuum.

Proof. $X$ is compact Hausdorff and $F: X \rightarrow S^{2}$ is monotone and onto. Thus, $F^{-1}\left(S^{2}\right)=X$ is a continuum since $S^{2}$ is.

Lemma 4. Given any isometry (rigid rotation) $g: S^{2} \rightarrow S^{2}$, there is a unique homeomorphism $h \in H(X)$ such that the foilowing diagram commutes:

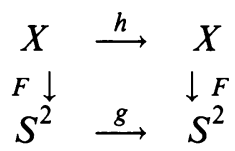

and $h$ is given by $h(x, y)=(g(x), g(y))$.

Proof. The map $h$, as defined, is clearly a homeomorphism of $X$ since $g$ preserves perpendicularity and $h(B(x, U, \varepsilon))=B(g(x), g(U), \varepsilon)$, so that $h$ (and similarly $h^{-1}$ ) takes basic open sets to basic open sets. Suppose $H: X \rightarrow$ $X$ is another homeomorphism such that the above diagram commutes; that is, $F \circ H=g \circ F$. If $H \neq h$, then there exists a nonempty open set $U \subset X$ such that $H(U) \cap h(U)=\varnothing$. Since $U$ contains a saturated open set $V \neq \varnothing$, there exists $x \in S^{2}$ such that $\{x\} \times S^{1}(x) \subset U$. Thus, $H\left(\{x\} \times S^{1}(x)\right) \cap$ $h\left(\{x\} \times S^{1}(x)\right)=\varnothing$. But $F\left(h\left(\{x\} \times S^{1}(x)\right)\right)=g\left(F\left(\{x\} \times S^{1}(x)\right)\right)=\{g(x)\}$, so $F\left(H\left(\{x\} \times S^{1}(x)\right)\right)=\{g(x)\}$ also. However, this means that $H\left(\{x\} \times S^{1}(x)\right) \subset$ $F^{-1}(\{g(x)\})$; that is $H\left(\{x\} \times S^{1}(x)\right) \subset\{g(x)\} \times S^{1}(g(x))=h\left(\{x\} \times S^{1}(x)\right)$ contradicting the fact that $H\left(\{x\} \times S^{1}(x)\right) \cap h\left(\{x\} \times S^{1}(x)\right)=\varnothing$ and completing the proof.

It is now easy to prove the next result. 
Theorem 1. $X$ is homogeneous.

Proof. Let $(x, y)$ and $(p, q) \in X$. Then $x \perp y$ and $p \perp q$. There is a rigid rotation $g$ of $S^{2}$ such that $g(x)=p$ and $g(y)=q$. (First rotate $x$ to $p$ with a rotation $g_{2}$. Then using $p$ as the center of rotation, rotate $g_{2}(y)$ around to $q$. Call this rotation $g_{1}$. Such a $g_{1}$ exists since both $g_{2}(y)$ and $q$ lie on $S^{1}(p)$. Then $g=g_{1} \circ g_{2}$ is the desired rotation.) The homeomorphism $h$ of $X$ associated with $g$, as in Lemma 4 , has the property that $h(x, y)=(p, q)$, and the proof is done.

The next result gives us what we need to prove that $X$ does not have the Effros property and is thus a counterexample to Ancel's two questions.

Lemma 5. For every $x \in S^{2}$ and homeomorphism $h \in H(X), h\left(\{x\} \times S^{1}(x)\right)=$ $\{y\} \times S^{1}(y)$ for some $y \in S^{2}$.

Proof. $h\left(\{x\} \times S^{1}(x)\right)$ is a simple closed curve, and $X \backslash\left(h\left(\{x\} \times S^{1}(x)\right)\right)$ is connected since $X \backslash\left(\{x\} \times S^{1}(x)\right)$ is connected. Suppose $h\left(\{x\} \times S^{1}(x)\right)$ is not equal to any $\{y\} \times S^{1}(y)$. Then, $h\left(\{x\} \times S^{1}(x)\right)$ cannot be contained in any single $\{y\} \times S^{1}(y)$ either, because no simple closed curve properly contains another one. Thus $F\left(h\left(\{x\} \times S^{1}(x)\right)\right.$ is a nondegenerate continuum in $S^{2}$. If $h\left(\{x\} \times S^{1}(x)\right) \cap\left(\{y\} \times S^{1}(y)\right)$ is connected (or empty) for every $y \in S^{2}$, then $F\left(h\left(\{x\} \times S^{1}(x)\right)\right)$ is a simple closed curve and so separates $S^{2}$. On the other hand, if $h\left(\{x\} \times S^{1}(x)\right) \cap\left(\{p\} \times S^{1}(p)\right)$ is disconnected for some $p$, then $F_{p}\left(h\left(\{x\} \times S^{1}(x)\right)\right.$ (see Lemma 2$)$ is a subcontinuum of a disk $D=F_{p}(X)$ that intersects the boundary of $D$ in a disconnected set. Thus by [5, Theorem 28, p. 156], $F_{p}\left(h\left(\{x\} \times S^{1}(x)\right)\right)$ separates the disk. By shrinking the boundary of $D$ to a point, we get that $F\left(h\left(\{x\} \times S^{1}(x)\right)\right)$ separates $S^{2}$ in this case also. $\left(F=s_{p} \circ F_{p}\right.$ where $s_{p}$ shrinks just the set $\{p\} \times S^{1}(p)$ to a point; $F_{p}$ has already shrunken every $\{x\} \times S^{1}(x)$ to a point for $x \neq p$.)

Since $F\left(h\left(\{x\} \times S^{1}(x)\right)\right)$ separates $S^{2}$, we can choose $a, b$ belonging to different components of $S^{2} \backslash F\left(h\left(\{x\} \times S^{1}(x)\right)\right)$. For every $(p, q) \in M=$ $X \backslash\left[h\left(\{x\} \times S^{1}(x)\right) \cup F^{-1}(\{a, b\})\right]$, there exists a basic open set $B[p, q]$ containing $(p, q)$ and contained in the open set $M \subset X$. By an easy compactness argument there is an $\varepsilon>0$ such that $B\left(a, S^{1}(a), \varepsilon\right) \cap h\left(\{x\} \times S^{1}(x)\right)=\varnothing$, $B\left(b, S^{1}(b), \varepsilon\right) \cap h\left(\{x\} \times S^{1}(x)\right)=\varnothing$, and $B\left(a, S^{1}(a), \varepsilon\right) \cap B\left(b, S^{1}(b), \varepsilon\right)=\varnothing$. Then the collection $\left\{B\left(a, S^{1}(a), \varepsilon\right), B\left(b, S^{1}(b), \varepsilon\right)\right\} \cup\{B[p, q]:(p, q) \in M\}$ is an open cover of a connected set $M$. Hence, by the simple chaining theorem, there is a simple chain $\left\{U_{j}\right\}_{j=1}^{m}$ from this cover with $U_{1}=B\left(a, S^{1}(a), \varepsilon\right)$ and $U_{m}=B\left(b, S^{1}(b), \varepsilon\right)$. For each $j$ let $V_{j}$ be the saturated part of $U_{j}$. Notice that both $U_{j}$ and $V_{j}$ are connected for each $j$. Then $V_{1}=U_{1}$ and $V_{m}=U_{m}$. Furthermore, for each $j<m, V_{j+1}$ is dense in $U_{j+1}$, and thus $U_{j} \cap V_{j+1} \neq \varnothing$ since $U_{j} \cap U_{j+1} \neq \varnothing$. But since $V_{j+1}$ is open and $V_{j}$ is dense in $U_{j}$, we have $V_{j} \cap V_{j+1} \neq \varnothing$. Hence $\left\{V_{j}\right\}_{j=1}^{m}$ is a simple chain of saturated connected open 
sets joining $\{a\} \times S^{1}(a) \subset V_{1}$ to $\{b\} \times S^{1}(b) \subset V_{m}$. Thus $\left\{F\left(V_{j}\right)\right\}_{j=1}^{m}$ is a chain of connected open sets in $S^{2}$ joining $a$ to $b$, and missing $F\left(h\left(\{x\} \times S^{1}(x)\right)\right)$. This is impossible since $F\left(h\left(\{x\} \times S^{1}(x)\right)\right)$ separates $a$ from $b$, and this is a contradiction. Hence, we are done.

Theorem 2. The map $E_{(x, p)}: H(X) \rightarrow X$ defined by $E_{(x, p)}(h)=h(x, p)$ is not an open mapping, with $H(X)$ having the compact-open topology.

Proof. Let $(x, p) \in X$ and let $q \in S^{1}(x)$ with $q \neq p$. Let $U, V$ be disjoint open sets in $S^{1}(x)$ with $p \in U$ and $q \in V$. Then $B(x, U, 1)$ and $B(x, V, 1)$ are disjoint open sets containing $(x, p)$ and $(x, q)$ respectively. Let $O \subset$ $H(X)$ denote the set $\{h \in H(X): h(x, p) \in B(x, U, 1)$ and $h(x, q) \in$ $B(x, V, 1)\}$. This is a neighborhood of the identity in the compact-open topology. Then for $k \in O$ if $k(x, p) \notin\{x\} \times S^{1}(x)$ it follows that $k(x, p)$ belongs to the saturated part of $B(x, U, 1)$. But then $k(x, q) \in B(x, U, 1)$ also, which is a contradiction since $k(x, q) \in B(x, V, 1)$. Thus, $k(x, p) \in$ $\{x\} \times S^{1}(x)$, so that $E_{(x, p)}(O) \subset\{x\} \times S^{1}(x)$. Since $\{x\} \times S^{1}(x)$ is nowhere dense in $X, E_{(x, p)}(O)$ is not open, and the proof is complete.

We close with a question. A space $Y$ is two-homogeneous provided that given points $a, b, c, d \in Y$ with $a \neq b$ and $c \neq d$, there exists $h \in H(Y)$ such that $h(\{a, b\})=\{c, d\}$. Our space $X$ is not two-homogeneous. Do Ancel's questions have affirmative answers when $X$ is assumed to be two-homogeneous?

\section{REFERENCES}

1. F. D. Ancel, An alternative proof and applications of a theorem of E. G. Effros, Michigan Math. J. 34 (1987), 39-55.

2. R. F. Arens, Topologies for homeomorphism groups, Amer. J. Math. 68 (1946), 593-610.

3. __ A topology for spaces of transformations, Ann. of Math. (2) 47 (1946), 480-495.

4. E. G. Effros, Transformation groups and $C^{*}$-algebras, Ann. of Math. (2) 81 (1965), 38-55.

5. R. L. Moore, Foundations of point set theory, Amer. Math. Soc. Colloq. Publ., vol. XIII, rev. ed., Amer. Math. Soc., Providence, RI, 1962.

6. K. F. Porter, Evaluation maps on groups on self-homeomorphisms, Ph.D. Dissertation, University of Delaware, 1987.

Department of Mathematical Sciences, University of Delaware, Newark, Delaware 19716

Department of Mathematical Sciences, Saint Mary's College, Moraga, California 94575 